ESJ Social Sciences

\title{
Les Politiques Publiques De Gestion Des Dechets Au Cameroun : Une Analyse Critique Du 'Monopole' D'hysacam A L'ere De La Gouvernance Decentralisee Dans La Ville De Yaounde
}

William Hermann Arrey, Ph.D.

Chargé de Cours, Chef du Département de Paix et Développement Faculté des Sciences Sociales et des Relations Internationales (FSSRI), Université Protestante d'Afrique Centrale (UPAC), Yaoundé, Cameroun Alain-Patrick Loumou Mondoleba

Doctorant en Science Politique, Université de Douala, Douala, Cameroun

Doi:10.19044/esj.2021.v17n15p430

Submitted: 02 April 2021

Accepted: 04 May 2021

Published: 31 May 2021
Copyright 2021 Author(s)

Under Creative Commons BY-NC-ND

4.0 OPEN ACCESS

Cite As:

Arrey W.H. \& Loumou Mondoleba A-P. (2021). Les Politiques Publiques De Gestion Des Dechets Au Cameroun : Une Analyse Critique Du 'Monopole' D'hysacam A L'ere De La Gouvernance Decentralisee Dans La Ville De Yaounde.

European Scientific Journal, ESJ, 17(15), 430. https://doi.org/10.19044/esj.2021.v17n15p430

\section{Résumé}

A mesure que le Cameroun se modernise, la question des déchets dans la ville de Yaoundé occupe une place importante dans l'agenda des élites politico-dirigeantes. La croissance démographique rapide et l'industrialisation croissante sont productrices d'une quantité débordante des déchets dont les effets sur la santé et la sécurité des populations se font ressentir. Cet article se veut une réflexion critique du monopole octroyé à HYSACAM dans la gestion technique des déchets dans la ville de Yaoundé. Sur le plan méthodologique, cette réflexion s'appuie sur la recherche documentaire, l'investigation auprès des institutions directement concernées et sur un protocole d'enquête essentiellement qualitative dressé aux populations de Yaoundé. En s'encrant sur l'incrémentalisme, elle met en exergue le tâtonnement et le bricolage qui caractérisent la politique de gestion des déchets dans la ville de Yaoundé. Dit autrement, la politique de gestion des déchets dans la ville de Yaoundé s'inscrit dans un schéma de débrouillardise et de tâtonnement permanent, se caractérisant par des va-et-vient et des essais-recommencements infinis. Cette 
étude postule qu'on ne saurait envisager, en plein essor de la gouvernance décentralisée, une politique de gestion efficace des déchets en faisant abstraction au " marché » des partenaires techniques, qui suppose une démonopolisation d'HYSACAM dans la gestion technique des déchets dans la ville de Yaoundé.

Mots-clés: Gestion Des Déchets, Gouvernance Décentralisée, HYSACAM, Politiques Publiques, Yaoundé

\title{
Public Waste Management Policies In Cameroon: A Critical Analysis Of The Hysacam 'Monopoly' In The Era Of Decentralized Governance In The City Of Yaounde
}

\author{
William Hermann Arrey, Ph.D.
}

Senior Lecturer and Chair-Department of Peace and Development Studies, Faculty of Sciences in International Relations (FSSIR) Protestant University of Central Africa, Yaoundé, Cameroon

\section{Alain-Patrick Loumou Mondoleba}

Ph.D candidate in Political Science, University of Douala, Douala, Cameroon

\begin{abstract}
As Cameroon modernizes, the issue of waste in the city of Yaoundé occupies an important place on the agenda of the political-ruling elites. Rapid population growth and increasing industrialization are producing an overflowing quantity of waste, the effects of which are felt on the health and safety of populations. This article is a critical analysis of the monopoly granted to HYSACAM in the technical management of waste in the city of Yaoundé. Methodologically, the research is based on documentary analysis, investigation of the institutions directly concerned and on an essentially qualitative survey protocol drawn up to the populations of Yaoundé. By focusing on the public policy theory of incrementalism, it highlights the trial and error and tinkering that characterize the waste management policy in the city of Yaoundé. In other words, the waste management policy in the city of Yaoundé is part of a scheme of resourcefulness and permanent groping, characterized by back and forth and endless re-starts. This study argues that, in the boom of decentralized governance, an effective waste management policy cannot be envisaged by disregarding the "market" of technical partners, which presupposes a demonopolization of HYSACAM in the technical management of waste in the city of Yaoundé.
\end{abstract}


Keywords: Decentralized Governance, HYSACAM, Public Policies, Waste Management Yaoundé.

\section{Introduction}

La question de l'assainissement et de l'aménagement du territoire urbain se pose avec acuité à mesure qu'augmentent la démographie et l'espace urbains. Au sens d'Henri-Michel Obe et Nicolas Brou Ahossi (2019), « cette évolution de la population, exceptionnelle par son ampleur et sa rapidité, s'accompagne de dynamiques spatiales nouvelles, tant en termes de migration de population que d'urbanisation » (p. 21). Cette situation suscite un intérêt à s'intéresser aux politiques publiques de gestion des déchets et de jeter un regard sur «le marché » qui se crée autour de ceux-ci. Comme le souligne Giorgio Blundo (2009), « au-delà des implications évidentes sur la qualité de vie et sur les conditions sanitaires des citadins, l'hygiène et la salubrité urbaines représentent une entrée privilégiée pour l'analyse de la gouvernance locale » (p.113). Le marché de gestion des déchets au Cameroun donne à observer l'imbrication de deux dimensions : la dimension technique et la dimension politique. La dimension technique fait référence aux procédés de choix des «industries » de réassemblage, traitement, d'évacuation et de recyclage des déchets en fonction notamment des dispositifs technicomatériels (charrettes, bennes de ramassage et d'évacuation des déchets, engin de recyclage etc.) dont ils possèdent et de leur expérience en la matière. Elle renseigne également sur les technologies institutionnelles telles qu'elles se déploient. La dimension politique implique les jeux de pouvoir et d'intérêts des acteurs politiques impliqués dans la gestion des déchets, porteurs d'agendas divers. Elle permet de lire et de comprendre le pourquoi et le comment des dissonances compétencielles et des pesanteurs opérationnelles souvent observées. En effet, comme partout ailleurs, la gestion des déchets au Cameroun donne lieu à un véritable marché public dont le processus d'attribution laisse observer une circulation fluide de ressources financières et politiques (Maccaglia \& Cirelli, 2019).

L'on a souvent rappelé que l'une des raisons d'être des politiques publiques est de mettre de l'ordre et en ordre une société du désordre. Elle concilie par-là, l'ordre et le désordre en sonnant le glas de leur dialectique (Freund, 1986). En soi, la fabrique des politiques publiques constitue en effet " une activité politique nécessaire qui enchante le monde en montrant que les problèmes de société sont solubles » (Zittoun, 2013, p. 321). Le problème de l'invasion des déchets dans les zones urbaines au Cameroun est soluble par le biais des politiques publiques efficaces. La question des déchets et d'assainissement de manière générale est l'un des défis majeurs des élites politiques locales, qui s'attèlent - même si timidement en contexte camerounais - à "faire de 'l'ordre hygiénique" un enjeu de contrôle de 
l'espace public et qui jouent souvent, dans cette entreprise, une partie de leur crédibilité et légitimité »(V. Blundo, Ibid., p. 113). Elle est d’autant plus préoccupante qu'elle est étroitement liée à la santé des citoyens, du sol et à l'environnement. Analyser les politiques publiques de gestion des déchets c'est penser les modalités à partir desquelles l'Etat se fait du souci pour ses populations, les prend en souci (le souci populationnel) (Machikou, 2010, p.126) à l'effet de leur donner santé et vie. A partir de là, on voit s'établir une corrélation étroite entre les notions de biopouvoir et de biopolitique chères à Foucault $(1975$; 1997) et l'analyse des politiques de gestion des déchets.

La littérature sur la question des déchets en sciences sociales aborde la question suivant diverses perspectives. D'abord sous le prisme des politiques environnementales, cette perspective regroupe les travaux sur les initiatives locales relatives à la promotion «des formes innovantes et alternatives de traitement des déchets (...), ainsi que les travaux s'intéressant aux dimensions sociales des activités de récupération»(V. Maccaglia \& Cirelli, Ibid.). Ensuite, la perspective qui rassemble des travaux s'employant à démontrer que l'installation ou l'exploitation des équipements de traitement des déchets a souvent donné lieu à des conflits entre les citoyens et la société civil désireux de s'impliquer, si non de prendre en charge la gestion des déchets et les décideurs publics qui les confient plutôt aux industriels et aux experts. La technicité (en termes de dispositifs technologiques et mécaniques) et la complexité du problème contraignent l'Etat à se tourner vers des partenaires externes ou privés et à sous-traiter avec eux pour essayer de le résoudre. C'est le cas dans la ville de Yaoundé avec la société Hygiène et Salubrité du Cameroun (HYSACAM). Enfin, la perspective visant à établir le rapport des populations aux déchets et « les enjeux éthiques de leur prise en charge (...). Cette approche interroge tout particulièrement la dimension éthique et «les jeux de caractérisation morale » de la promotion des pratiques de tri et de recyclage en mettant en lumière le caractère politique du déchet (Ortar \& Anstett, 2017). Cet article questionne le choix des partenaires et des instruments et querelle la centralisation et monopolisation de la gestion des déchets dans la ville de Yaoundé par HYSACAM, là où la gouvernance appelle à une décentralisation de celle-ci par la diversification des partenaires techniques.

En effet, de nombreuses initiatives vont être prises par le gouvernement pour tenter de résoudre le problème de l'insalubrité dans la ville de Yaoundé. En 2004, il sera mis en place le Comité interministériel de gestion des déchets, un organe mixte dont le rôle est de prendre les décisions, planifier et coordonner les projets sur les déchets. C'est à HYSACAM que reviendra l'exclusivité concessionnaire de gestion des déchets dans la ville de Yaoundé. De nombreux travaux s'attèleront à mettre à nu les difficultés auxquelles un tel monopole a conduit (Sidi Ould, 2006 ; Ademe, 2004 ; Enda Malgreb, 2003 
; Matejka, 2005). On notera par exemple des insuffisances institutionnelles, organisationnelles, matérielles et financières qui ont pour corollaire l'inefficacité de la politique de gestion des déchets dans la ville de Yaoundé. Entre 1968 et 1993 HYSACAM n'arrive à desservir que le centre-ville et les grands axes (Ngambi, 2015). Cet article passe en revue, et ce de manière critique, les politiques publiques de gestion des déchets au Cameroun en mettant un accent sur ce rôle monopolistique d'HYSACAM dans la ville de Yaoundé. Il débouche sur un examen prescriptif en proposant au gouvernement, à partir d'une perspective de la gouvernance décentralisée et du partenariat, des éléments d'ajustement de la politique publique de gestion des déchets existante dans la ville de Yaoundé.

Ainsi, après avoir décliné les éléments méthodologiques et théoriques sur lesquels cette réflexion s'appuie, l'analyse s'emploie à examiner d'abord le monopole d'HYSACAM dans la gestion technique des déchets dans la ville de Yaoundé, comme une logique antipodique du marché et de la gouvernance publique. Ensuite, elle établit le constat que la politique de gestion des déchets dans la ville de Yaoundé est une politique incrémentale et rationnellement limitée, et débouche enfin sur la possible réinvention de celle-ci en mettant en exergue le rôle de la gouvernance décentralisée.

\section{Cadre théorique et méthodologique de l'étude \\ Le lieu de l'étude}

Yaoundé, capitale politique du Cameroun est peuplée d'environnement de 4100000 d'habitants en 2019. Constituée de sept arrondissements (Yaoundé 1 (Nlongkak) ; Yaoundé 2 (Tsinga); Yaoundé 3 (Efoulan) ; Yaoundé 4 (Kondengui) ; Yaoundé 5 (Essos) ; Yaoundé 6 (BiyemAssi) et Yaoundé 7 (Nkolbisson)), la ville s'étend sur $304 \mathrm{~km}^{2}$ dont une superficie urbanisée de $183 \mathrm{~km}^{2}$.

\section{Le socle théorique de la réflexion : l'incrémentalisme}

Cet article s'appuie théoriquement sur l'incrémentalisme. Souvent associé aux travaux de Charles Lindblom (1979), l'incrémentalisme dans l'analyse des politiques publiques tend à montrer que «les décideurs politiques réalisent généralement des changements de l'action publique qui ne modifient que marginalement la situation en cours ou le statu quo ». Ils n'accordent pas une place importante au « changement radical », à l'évolution de la situation, encore moins à «toute réforme substantielle des politiques préalablement menées (...). Les décisions prises provoquent plutôt des petits ajustements marginaux, ou incrémentaux, qui visent à améliorer une action publique existante, sans réellement la remettre en question » (Jönsson, 2010, p. 317). Les ajustements en questions résultent des tâtonnements et d'une multiplicité des négociations à petite échelle qui font intervenir diverses 
rationalités. En se nourrissant des travaux d'Herbert Simon (1945) sur la rationalité limitée, l'incrémentalisme donne à voir comment le monopole technique octroyé à HYSACAM dans la gestion des déchets dans la ville de Yaoundé ne permet pas d'aboutir à une politique efficace ou optimale de gestion des déchets dans ladite ville.

\section{Description de la démarche méthodologique : techniques de collecte des données sur le terrain, échantillon et durée d'investigation}

Sur le plan méthodologique, cette réflexion s’appuie sur la recherche documentaire, l'investigation auprès des institutions directement concernées (Communauté urbaine de Yaoundé, l'ensemble des sept mairies de Yaoundé, le ministère de l'habitat et du développement urbain (MINHDU), HYSACAM-Yaoundé) et sur un protocole d'enquête essentiellement qualitative dressé aux populations de Yaoundé. L'étude a retenu un échantillon de dix ménages (10) par commune, soit soixante-et-dix (70) ménages au total. Il s'agissait d'évaluer l'appréciation de la politique de gestion des déchets par les populations. Le travail de terrain s'est étalé sur une période de dix (10) jours, soit du 14 novembre au 24 novembre 2020. On le voit donc, la démarche de vérification des données qui a été usitée est l'enquête informatique. Celle-ci se ramène à « l'observation systématique, la théorisation et la détermination des faits " (Gauthier, 2003, p. 16). La recherche documentaire a permis de prendre connaissance des travaux existant en la matière et d'engraisser notre socle analytique. Elle a reposé sur l'examen de tous les documents fournis par les différents acteurs directement impliqués dans l'élaboration et la mise en œuvre de la politique publique de gestion des déchets dans la ville de Yaoundé (documents portant sur les missions, les plans d'action, la structure organisationnelle, le budget, les objectifs, les réalisations etc. et les différents rapports). L'analyse documentaire a également permis de conceptualiser et de compléter les informations recueillies lors des entretiens.

\section{Point général sur le 'monopole' d'HYSACAM dans la gestion technique des déchets dans la ville de Yaoundé : une logique antipodique du marché et de la gouvernance publique}

Le « desserrement du verrou de l'Etat » (Le Galès, 1999) au cours des années 1990 au Cameroun -notamment avec l'avènement des plans d'ajustements structurels - a donné lieu à la prédominance d'une logique de marché dans la gestion des affaires publiques de manière générale. Il a permis de passer d'une lecture 'top-down' des politiques publiques et du gouvernement des territoires, à une lecture 'button-up' guidée par le nouveau paradigme de la gouvernance. Certains ont d'ailleurs postulé la thèse d'un affaiblissement de l'Etat (Donzelot \& Estèbe, 1994) là où d'autres y voyaient sa transformation, voire sa recomposition (King \& Le Galès, 2011). Tout 
compte fait, le marché induit la concurrence et la compétitivité. Il est constitutif de la gouvernance qui elle suppose un élargissement et une diversification du schéma actanciel configurant l'élaboration et la mise en œuvre des politiques publiques. En effet, la gouvernance désigne le plus souvent «les formes contemporaines de régulation collective qui se développent sur la base de relations entre acteurs publics et privé, et dans des cadres institutionnels et territoriaux pluriels, plus ouverts et plus instables. On parle ainsi souvent de " gouvernance multi niveaux " pour signifier à quel point les problèmes publics sont de plus en plus traités par des acteurs divers, placés à différents échelons territoriaux » (Nay et al., 2004, p. 230). Contrairement à la notion de gouvernement qui laisse apparaître l'idée d'un pouvoir centralisé et monopolisé par l'Etat dans un cadre national, la gouvernance suppose une prise en compte d'une pluralité d'acteurs agissant collégialement dans les processus d'élaboration, de prise de décision et de même de mise en œuvre. La présente réflexion s'efforce de mettre en relief HYSACAM en tant qu'instrument d'action publique, le public (les populations de la ville de Yaoundé) et la salubrité urbaine.

En effet, la gestion des déchets dans la ville de Yaoundé commence dès les années 1960, et jusqu'en 1968, la ville ne dispose qu'une section d'enlèvement des ordures ménagères qui ne s'occupera que d'un linéaire d'environ 100 kilomètres. Ladite section sera constituée de 36 employés et disposera que de trois véhicules. La décharge est encore située dans un quartier de la ville appelé Oyomabang, soit à environ trois kilomètres du centre-ville (Bessala Ngoma, 2003). Une bonne quantité de déchets est déversée dans les brousses, les bordures de routes, les alentours des habitations. En partie, cette situation s'explique par le fait que la ville était encore modeste, moins dense et peuplée qu'aujourd'hui. Très rapidement, la ville va se moderniser avec une plus grande industrialisation et s'agrandir démographiquement. La quantité des déchets produits par jour va considérablement augmenter. Le problème lié à la gestion des déchets va se poser avec une plus grande acuité, contraignant les autorités publiques à se tourner vers de partenaires techniques spécialisés en la matière. Ainsi, de 1968 à 1993, la Communauté urbaine de Yaoundé va concéder la gestion des déchets à la société Hygiène et Salubrité du Cameroun (HYSACAM) pour un contrat de trois ans qui sera renouvelé à chaque fois. Au cours de ces années, le service d'HYSACAM ne bénéficiera qu'au centreville et aux grands axes de la ville de Yaoundé. Les « sous-quartiers » ne sont quasiment pas desservis. A l'intérieur de ces derniers, les habitants créent incessamment des dépotoirs de déchets, en l'absence des bacs à ordures. D’après les estimations, «la quantité d'ordures ménagères collectée quotidiennement par HYSACAM varie entre 400 et 500 tonnes ; soit une moyenne d'environ 40\% de la production seulement " (Environnement Recherche Action au Cameroun, 2015, p. 10). 
Entre 1992 et 1998, l'Etat va engager un processus de rationalisation de la politique de gestion des déchets dans la ville de Yaoundé en octroyant une concession de plus à une autre société privée d'hygiène: Services Camerounais d'Assainissement (SECA). C'est une société anonyme de droit camerounais exerçant sur toute l'étendue du territoire. Il a pour mission principale de desservir le secteur industriel. Nombre de personnes rencontrées estiment que le contrat concédé au SECA avait une visée beaucoup plus politique que sociale. Il intervient à une période où le Cameroun s'apprêtait à organiser ses premières élections présidentielles pluralistes. L’insalubrité dans la ville faisant partie des préoccupations quotidiennes des populations de Yaoundé, le Président prit l'initiative de signer un contrat de 850 millions de FCFA avec le SECA le 03 janvier 1992 pour l'enlèvement de 68000 tonnes d'ordures. Les données collectées font état de ce que le SECA disposait de 250 employés, onze (11) camions à compaction, six (06) multi-bennes, trois (03) ampliroll, trois (03) grues, vingt (20) coffres de $16 \mathrm{~m} 3,100$ coffres de $6 \mathrm{~m} 3$ et 100 conteneurs de 1m3 (Ngambi, 2015, p. 62). De plus, le SECA bénéficiait d'un appui de 60 camions de 10 tonnes d'HYSACAM en provenance de Douala (Ibid., p. 63). Le contrat du SECA arrive à son terme en juillet 1992 avec des factures impayées. Cette rupture va pousser les différentes Communes de Yaoundé à reprendre la gestion des déchets. Un autre contrat sera négocié et signé en 1993 avec la société privée Dragages-Cameroun qui sera aussitôt (à la fin de la même année) rompu pour manque de moyens financiers (Ibid., p. 65). Le départ de Dragages-Cameroun ouvre la porte à un retour de la société privée CAMECAM qui fera elle aussi long feu pour faute d'expérience technique, d'équipements techniques et de manque de personnel qualifié. Ces lacunes techniques et opérationnelles vont contraindre la société à mettre fin à ses services dès la fin de la même année. A nouveau, l'insalubrité sévit dans la ville. On peut le voir, au lieu de procéder à une multiplication des partenaires techniques, les autorités se sont contentées de les succéder sans pour autant les multiplier et les faire co-agir. Une co-action de plusieurs partenaires techniques aurait concouru à rendre la politique de gestion des déchets dans la ville de Yaoundé plus efficace.

Il y'a donc un rebondissement irréversible d'HYSACAM qui se fait observé. La politique de gestion des déchets dans la ville de Yaoundé s’inscrit dans un schéma de débrouillardise et de tâtonnement permanent, se caractérisant par des va-et-vient et des essais-recommencements infinis. On dirait que les autorités politiques sont en carence de partenaires techniques avec lesquels elles peuvent collaborer. Elles évoquent comme principale raison de cette débrouillardise, le déficit des moyens financiers. Le financement octroyé au gouvernement camerounais par la Banque Mondiale pour assainir la ville de Yaoundé en 1994 permettra la mise sur pied du Programme Social d'Urgence (PSU). Le PSU regroupera des ONG, des 
Associations de quartier et des Groupements d'initiative commune (GIC) dont l'expérience et les compétences en matière de gestion des déchets seront très limitée. En 1997, c'est-à-dire trois ans plus tard, le PSU va fermer ses portes. Sa fermeture n'induira pas la fin des activités des ONG, GIC et Associations qui collaboraient avec lui. Ceux-ci vont poursuivre leurs missions ramassage et recyclage des déchets, même si faisant face à des contraintes matérielles et financières.

Cette situation va à nouveau pousser les autorités publiques à signer un contrat de concession avec HYSACAM, lequel contrat court depuis 1998. Ce retour vers HYSACAM est une preuve que les autorités de la Communauté urbaine de Yaoundé n'ont pas pu trouver une solution adéquate au problème d'insalubrité dans la ville de Yaoundé malgré la volonté et les multiples tentatives d'amélioration et/ou de rationalisation. Au départ, le contrat avec HYSACAM est de trois ans renouvelable. A cet effet, il a été renouvelé trois fois. Après les 9 ans d'activités, HYSACAM va signer avec la Communauté urbaine, un autre contrat d'un an (Ngnikam, 2013). Une fois que le contrat d'un an est arrivé à son terme (en 2007), la durée des contrats est passée de trois ans à cinq ans renouvelables. Cette prorogation des contrats est un indicateur de ce que les autorités publiques semblent se contenter du travail abattu par HYSACAM ; c'est par ailleurs un signe de l'évolution de degré de confiance qu'elles lui font. L'exclusivité du contrat de prestation accordée à HYSACAM « empêche d'emblée les autres acteurs de la société civile d'être impliqués dans les projets de gestion linéaire des déchets » (Ngambi, p. 125). Les autorités publiques brandissent l'argument de l'expérience et des compétences techniques du partenaire pour justifier son monopole dans toute la ville. L'échec des autres partenaires privés avec lesquels les autorités ont tenté d'opérer et de démonopoliser le marché au cours des années antérieures les conforte encore dans cette logique d'inchangeabilité. Une telle situation ne peut créer qu' " une condition de dépendance des pouvoirs publics vis-à-vis d'interlocuteurs qui disposent de disponibilités foncières et financières pour implanter des équipements de traitement. Différents contextes nationaux ont montré comment des acteurs privés ont constitué une situation de monopole de la gestion des déchets, notamment par une maîtrise du territoire et se sont imposés comme des interlocuteurs incontournables des autorités locales » (Maccaglia \& Cirelli, 2019, p. 2), et c'est le cas d'HYSACAM à Yaoundé.

\section{La politique de gestion des déchets dans la ville de Yaoundé : une politique incrémentale et rationnellement limitée aux déterminants et effets pluriels}

La politique incrémentale repose sur un système de décision dont le but est de modifier la situation sociale donnée de manière graduelle, « en ne considérant qu'un nombre limité, et donc non exhaustif, d'alternatives 
décisionnelles » (Bérard, 2011, p. 6). Les résultats issus de l’observation et des entretiens menés donnent à voir que la démarche adoptée par les autorités publiques dans la gestion des déchets est incrémentale et peut s'expliquer tant par des jeux et enjeux politiques que par les effets d'une rationalité limitée. Une politique incrémentale c'est une politique dont les objectifs ne sont pas suffisamment atteints ; c'est une politique dont les effets produits restent insatisfaisants pour le public cible. Les populations ne sont pas satisfaites de l'état actuel de la ville. HYSACAM ne pouvant pas desservir tous les coins de la ville, des populations ont elles-mêmes développé des moyens divers pour se débarrasser des déchets : le dépôt des ordures dans les rigoles, les égouts, les caniveaux, les lacs, l'entassement sur les chaussées, l'incinération à ciel ouvert. Ces pratiques sont devenues au sens de Bemb (2009), de véritables dangers dans la plupart des villes africaines comme est le cas de Yaoundé.

Les déterminants de cet incrémentalisme sont multiples et variés. Il s'agit entre autres du retrait financier de l'Etat (les subventions), de la défaillance du dispositif matériel des différentes communes d'arrondissement et des lacunes du cadre règlementaire local de la gestion des déchets ménagers (Ngambi, 2011). De plus, il convient de noter la faible prise en compte de la dimension psychosociologique des populations par la politique de gestion des déchets dans la ville de Yaoundé. En effet, Yaoundé est une ville cosmopolite constituée des populations venant de tous bords. Les villes africaines en générale ne se sont pas construites en rupture avec les mentalités villageoises. L'exode rural a provoqué un boom démographique des populations villageoises dans les villes. Les mentalités sont transposées des villages pour les villes. L'arrière des habitations s'érige sans gêne en dépotoir de déchets. Si dans les campagnes cela est vivable, en ville, l'arrière d'une habitation est la devanture d'une autre habitation. Dès lors, l'habitude qui vise à transformer le derrière de sa maison en dépotoir d'ordure ne saurait être vivable en ville. Ajouté à tout ceci, l'absence d'une réelle planification urbaine.

Dans les sous quartiers et/ou les quartiers desservis, les populations s'organisent à gérer elles-mêmes leurs déchets en mettant sur pieds des dispositifs des pré-collecte (Chalot, 1991). Une fois les déchets pré-collectés, ils sont ramassé par des individus qui en ont fait leur gagne-pain quotidien. Dans les quartiers comme Bonas, Ecole des postes, Obili, nous avons recensé deux catégories de ramasseurs: ceux qui disposent des brouettes ou 'poussepousses' et qui ont une période bien déterminée de leur passage dans les cités (une à deux fois par semaines), pour une paie dont la somme varie entre 2000 et 3000 CFA par mois, et ceux qui, généralement sillonnent dans les quartiers, en faisant le porte-à-porte à l'effet de vider directement les poubelles encombrantes au prix de 50-100 FCFA. Cette dernière catégorie est constituée d'enfants et d'adolescents dont la tranche d'âge varie entre 10 et 14 ans. Dans l'ensemble, leur rôle est d'emballer les déchets dans des vieux sacs 
et de les transporter jusqu'au niveau des bacs à ordures. Toutefois, il est à déplorer le fait que le plus souvent, ces agents pré-collecteurs reversent les ordures dans la rue ou aux bordures de la route plutôt que les évacuer dans les bacs à ordures ou les centres de groupages (Yao -Kouassi \& Gohourou, 2018). En effet, les enfants engagés comme pré-collecteurs transportent ces déchets vers ces lieux de dépôts n’ont pas suffisamment de taille et de force pour les jeter à l'intérieur du bac à ordures.

D’après une étude réalisée par Pélagie Yolande Epoh-Mvaboum et Isaac Moussinga (2019), près de 40\% de la population de Yaoundé n’ont pas accès au service de collecte de déchets fourni par HYSACAM. Ces populations n’hésitent ainsi pas à se servir des espaces inhabités les plus proches de leurs concessions pour en faire des dépotoirs de déchets. Cette pratique a des conséquences énormes tant sur l'environnement que sur la santé des populations qui y vivent. Les cours d'eau qui traversent la ville sont pollués, l'invasion des moustiques et autres insectes vecteurs de nombreuses maladies se fait observer. En contexte d'Afrique subsaharienne en générale, il s'agit des maladies telles que le paludisme comme souligné plus haut, des Infection Respiratoire Aigüe (IRA) et la diarrhée. Ces maladies représentent d'ailleurs 60\% des impacts connus de l'environnement sur la santé en Afrique (Koné-Bodou et al, 2020). L’incinération des déchets pratiquée par les populations est porteuse d'émission des gaz à effet de serre ayant un impact direct sur la couche d'ozone. Les déchets déversés dans les caniveaux et les cours d'eau les bouchent et engendre des inondations et des éboulements dans les quartiers situés dans les bas-fonds. En réalité, la pratique devenue courante dans la ville de Yaoundé de décharge hasardeuse des déchets provoque, non seulement des nuisances générées (odeurs, fumées, plastiques volants), mais aussi la pollution des sols et des eaux de la nappe phréatique (Biaou et al., 2019, p. 96). En effet, certains de ces déchets -même si parfois riches en matière organique - produisent des éléments indésirables pour la santé et la sécurité des populations. Or, un Etat qui se veut soucieux de la santé de ses populations c'est un Etat qui s’investit dans la prévention des écosystèmes dans lesquels vivent ces populations. En effet, il existe, à ne point nier, une corrélation entre la mauvaise gestion des déchets et la prévalence de certaines maladies. La ville de Yaoundé s'est souvent illustrée par son insalubrité (déchets déversés sur les bordures de routes, les eaux usées non canalisées, les voiries dégradées, l'insuffisance d'égouts, pollution d'air, etc.). Au cours des années 1994, les immondices non ramassées sont devenues un véritable cauchemar pour les habitants de la ville avec l'arrêt du ramassage des déchets (Ngambi, 2015 ; Gapyisi, 1989). La ville sera différemment qualifiée par des médias de : "Yaoundé, ville poubelle »; "Yaoundé l'agonie »; "Yaoundéla-sale » ; «Yaoundé l'affreuse » (Zoa, 1996), et ce phénomène est encore 
clairement visible dans la ville aujourd'hui. La photo ci-dessous en dit d'ailleurs long.

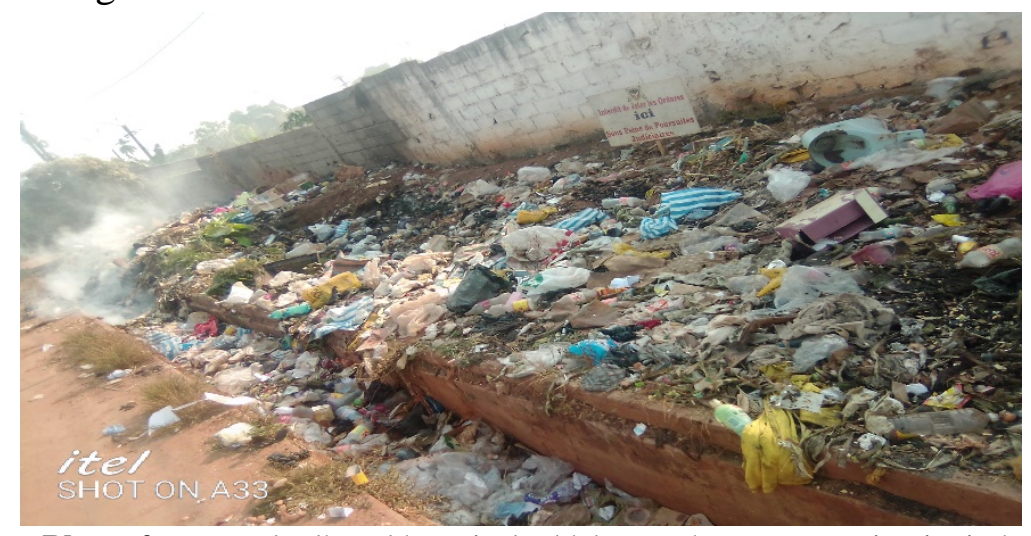

Photo 2. Exemple d’un dépotoir de déchets ménagers en peine incinération et bouchant les caniveaux initié par les populations du quartier estudiantin Bonas.

Source : (notre étude, 2020)

La gestion disproportionnelle des déchets par HYSACAM crée des frustrations et des sentiments d'inégalités chez les populations ne bénéficiant pas de ses services. En effet, le ressenti des populations de Yaoundé que nous avons enquêtées par rapport à la gestion des déchets varie en fonction de leur milieu d'habitation. La perception et la réception en dépendent également. Toutefois, 59 \% estiment insatisfaisants les services offerts par HYSACAM. Aussi convient-il de souligner que même dans les quartiers considérés comme étant des quartiers de résidence (Odza, Bastos) des populations se plaignent de la faible capacité d'HYSACAM à y mettre de la propreté. Les enjeux poussent peut-être à s'interroger sur l'état actuel de la gestion centralisée des déchets dans un contexte de décentralisation croissante au Cameroun qui pourtant tente d'attribuer un rôle important aux autorités locales élues (maires, maires de villes et présidents de conseil régional) et demande à mettre désormais les citoyens ordinaires au centre de leurs propres préoccupations de développement individuel et/ou collectif.

\section{Réinventer et ajuster la politique de gestion des déchets dans la ville de Yaoundé : le rôle de la gouvernance décentralisée.}

S'il est vrai que les questions d'hygiène et d'assainissement constituent des préoccupations anciennes, force est de reconnaitre que leur intérêt a connu un relief ces dernières années. Cette évolution est notamment tributaire des mutations du cadre législatif et réglementaire qui organise la prise en charge de ces questions. A ce sujet, la Loi N96/06 du 16 janvier 1996 portant révision de la constitution du 2 juin 1972 tient un rôle très important. En réaffirmant le caractère décentralisé de l'Etat unitaire du Cameroun, cette loi a doté les collectivités territoriales (la commune et la 
région) de pouvoirs particuliers, au nombre desquels figurent la prise en charge des questions d'hygiène et d'assainissement. En plus, la Loi $\mathrm{N}^{\circ}$ 2004/017 du Juillet 2004 d'orientation de la décentralisation stipule que : « La décentralisation constitue l'axe fondamental de promotion du développement, de la démocratie et de la bonne gouvernance au niveau local » (Article 2 alinéa 2).

En ce qui concerne l'hygiène, l'Etat a mis sur pied des dispositions et des lois régissant l'activité. Tout d'abord dans la Loi Nº 2004/018 du 22 juillet 2004 fixant les règles applicables aux communes, certaines dispositions donnent compétence aux communes. L'article 16 par exemple transfère aux communes la compétence de nettoyer les rues, chemins et espaces publics communaux ; d'assurer le suivi et le contrôle de la gestion des déchets industriels ; de lutter contre l'insalubrité, les pollutions et les nuisances ; d'élaborer les plans communaux d'action pour l'environnement et de gérer au niveau local des ordures ménagères. S'agissant de l'environnement, l'Etat a pris des mesures nécessaires pour sa protection. Dans les articles 1 et 2 du Décret N²012/0882/PM du 27 mars 2012 fixant les modalités d'exercice de certaines compétences transférées par l'Etat aux communes en matière d'environnement, il est assigné aux communes la tâche d'Élaborer des plans d'action pour l'environnement et de lutter contre l'insalubrité, les pollutions et les nuisances (article 1). De plus, il revient aux élus locaux d'élaborer et de mettre en œuvre la politique nationale en matière d'environnement et de développement durable (article 2).

De l'analyse des différentes dispositions normatives sus énoncées, il ressort clairement que l'élan actuel du processus de décentralisation au Cameroun donne à s'interroger sur la raison d'être de la monopolisation centralisée de HYSACAM dans la gestion technique des déchets dans la ville de Yaoundé. Un secteur dont les compétences et les ressources sont largement dévolues aux organes locaux (maires, conseillers municipaux, maire de ville, conseillers régionaux, si l'on s'en tient aux dispositions des instruments relatifs à la gouvernance locale et à la décentralisation. Par conséquent, il semble que toute solution à la gestion monopolisée des déchets dans la ville de Yaoundé et aux conséquences y afférentes réside dans le développement des politiques et des stratégies de décentralisation qui donneront aux maires des sept communes de Yaoundé la capacité financière, administrative et technique et assez d'autonomie pour gérer efficacement les déchets dans la ville, en collaboration avec les organisations de la société civile (ONG, CIG, etc.), le secteur privé et la population locale en tant que partenaires actifs. Il sera également nécessaire, dans le cadre de la mise en œuvre de la décentralisation actuelle, d'accorder une attention particulière à la coopération décentralisée. 
En claire, les autorité politiques locales doivent non seulement s'imprégner des compétences et des ressources qui leur sont transférées en matière de gestion des déchets, mais aussi, procéder à un décentrement, mieux, à une libéralisation des processus décisionnels y afférents. Il s'agit d'expérimenter un logiciel managérial qui encourage le marché, c'est-à- dire la collaboration, la coopération, le partenariat et la contractualisation. Cela suppose également une diversification des partenaires et donc, une démonopolisation de la gestion technique des déchets dans la ville de Yaoundé qui n'a jusqu'ici pas permis l'assainissement et l'urbanisation souhaitée dans ladite ville.

\section{Conclusion}

Jusqu'ici, le but de cet article a été de passer en revue, de manière critique, les politiques publiques de gestion des déchets au Cameroun en mettant l'accent sur le rôle monopolistique d'HYSACAM dans la ville de Yaoundé. Par ailleurs, l'intérêt a été porté sur les possibilités d'ajustement de ces politiques, ce qui a donné lieu à une réflexion sur des perspectives fondées sur des preuves que le gouvernement pourra mettre à profit afin d'améliorer ses politiques publiques de gestion des déchets. Cela devra passer, l'avonsnous martelé, par l'adoption d'une démarche qui s'inscrive dans le paradigme managérial de gouvernance décentralisée et de partenariat. La réflexion arrive donc à la conclusion que le monopole de gestion technique des déchets octroyé à HYSACAM n'est pas de nature à favoriser une gestion efficace des déchets dans la ville de Yaoundé car, les problèmes d'insalubrité et de pollution y demeurent avec une plus grande acuité. Cela induit un cadre de vie souillé par des odeurs nauséabondes, des maladies et la présence remarquable de moustiques, insectes, rats et mouches.

Dans une perspective plus large, il est essentiel de souligner que, bien que l'analyse connaisse certaines limites par rapport aux données (par exemple, la portée de l'étude se concentrant uniquement sur Yaoundé et l'utilisation limitée d'enquêtes qualitatives en raison de facteurs financiers, temporels et humains, contraintes de ressources), elle a néanmoins produit des enseignements utiles à la gestion des déchets dans la ville de Yaoundé. Les diagnostics qu'elle pose pourront servir à améliorer la gestion des déchets dans d'autres villes métropolitaines comme Douala (la capitale économique), qui sont tout aussi soumises aux contraintes structurelles et opérationnelles liées à la gestion des déchets et où HYSACAM détient le monopole. À plusieurs égards, cet article a également fourni une plate-forme pour les recherches futures de cette catégorie dans des contextes aussi complexes que celui du Cameroun avec cependant peu de recherches empiriques réalisées et en cours [de réalisation] sur la forte monopolisation de la gestion technique des déchets à l'ère de la décentralisation. Pour la raison que les autorités politiques locales 
élues opèrent dans un environnement polycentrique composé de multiples lignes de revendications des responsabilités hiérarchiques et où le gouvernement local fort et la citoyenneté active doivent cohabiter et trouver leur chemin dans le processus de décentralisation du Cameroun, cette étude sera judicieux pour les recherches futures sur la question. Elle leur permettra d'examiner les circonstances dans lesquelles la gouvernance locale polycentrique est construite et quels espaces s'ouvrent au renforcement de l'administration locale et de la citoyenneté démocratique dans la conception des politiques publiques en général (Arrey, 2011), et les processus de mise en œuvre des politiques de gestion des déchets en particulier.

Toutefois, il faut reconnaître les difficultés auxquelles les autorités pourraient faire face dans l'opérationnalisation des recommandations sus évoquées. Ces obstacles se résument pour l'essentiel aux difficultés financières (diversifier les partenaires techniques exige de disposer assez de ressources financières et cela s'avère périlleux pour les collectivités locales en contexte de non effectivité du transfert des ressources) et aux difficultés liées à l'indisponibilité des partenaires techniques qualifiés. Les tentatives de démonopolisation foirées survenues dans les années 1993 en disent long à propos. Les autorités devront donc sélectionner avec beaucoup de précautions les futurs nouveaux partenaires techniques.

\section{References:}

1. Arrey, W.H. (2011). Decentralisation as a Mechanism for Deepening Democracy in Contemporary Africa: An Investigation of the Case of Cameroon. Unpublished Ph.D Thesis. University of Bradford, U.K.

2. Bemb, C.G. (2009). Le traitement des ordures ménagères et l'agriculture urbaine et périurbaine dans la ville de Bertoua. Mémoire de master en Conseil général. Institut National de la Jeunesse et des Sports (INJS), Yaoundé.

3. Bérard, C. (2011). La démarche décisionnelle dans les systèmes complexes : incrémentalisme et rationalités. AIMS, Nantes, 1-24.

4. Bessala Ngoma, (2003). Collectivités locales et gestion déléguée des déchets : cas de Yaoundé au Cameroun. Yaoundé, Hysacam.

5. Bjørn Olsen, H. (2017). Décentralisation et gouvernance locale. Module 1: définitions et concepts », novembre 2017.

6. Blundo, G. (2009). Des ordures et des hommes : la gouvernance de l'assainissement à Dogondoutchi. In Tidjani Alou (Dir.). Les pouvoirs locaux au Niger. Tome I (pp. 113-127). Paris : Codesria-Karthala.

7. Biaou, I. C., Hedible, S. C., Codjo Landeou, R., \& Boko, M. (2019). Impact des Décharges Sauvages des Déchets Solides sur les Sols à Cotonou. European Scientific Journal. 15(30), 94-104. 
8. Chalot, F. (2004). De l'amont vers l'aval : l'émergence d'une filière de gestion des déchets adaptés aux villes africaines. Synthèse et analyse des actions relatives aux déchets. Gestion durable des déchets et l'assainissement urbain.

9. Drucker P. (1969). The age of Discontinuity. Guidelines to our changing society. New York: Harper and Row.

10. Environnement Recherche Action au Cameroun. (2002). Rapport final : Mise en place de structures de précollecte et de traitement des déchets solides urbains dans une capitale tropicale : cas de Yaoundé, Cameroun. Programme gestion durable des déchets et de l'assainissement urbain. Disponible sur : https://www.pseau.org/epa/gdda/Actions/Action_D05/Rapport_final_ D05.pdf.

11. Epoh-Mvaboum Y., \& Moussinga, I. N. (2019). La gestion des ordures ménagères et leur impact sur l'environnement : cas de la ville de Yaoundé. Consulté le 02 décembre 2020, sur https://www.sifee.org/static/uploaded/Files/ressources/actes-des colloques/ouagadougou/session-6/2_Moussinga_communication.pdf.

12. Foucault, M. (1976). Il faut défendre la société. Cours au Collège France 1976. Paris : Gallimard/Seuil.

13. Foucault, M. (1997). Surveiller et punir. Naissance de la prison. Paris : Gallimard, «Bibliothèque des histoires ».

14. Freund, J. (1986). L’essence du politique. Paris : Dalloz.

15. Gapyisi, E. (1989). Le défi urbain en Afrique. Paris : Harmathan.

16. Hirst, P. (2000). Democracy and Governance. In J. Pierre (Eds.). Debating Governance (pp. 13-35), Oxford: Oxford University Press.

17. Jönsson, A. (2010). Incrémentalisme. In L. Boussaguet (Eds.). Dictionnaire des politiques publiques: 3e édition actualisée et augmentée (pp. 317-325). Paris : Presses de Sciences Po

18. King, D., \& Le Galès P. (2011). Sociologie de l'État en recomposition. Revue française de sociologie. 52 (3), 453-480.

19. Koné-Bodou, P. J., Ballo Zié, Kouadio, A. S., Kouamé Kouamé, V., Yapi Dopé, A. C., \& Tidou Abiba S. (2020). Fardeau économique du paludisme et pratiques de gestion des ordures ménagères : cas des Communes de Cocody, Anyama, Port-Bouët, Et Yopougon (Abidjan, Côte d'Ivoire). European Scientific Journal. 16 (6), 152-167.

20. Le Galès, P. (1999). Le desserrement du verrou de l'État ? Revue internationale de politique comparée, 6 (3), 627-642.

21. Lindblom, C.E. (1979). Still muddling, not yet through. Public Administration Review, 39 (6), 517-526.

22. Maccaglia, F. et Cirelli, C. (2019). «Penser le politique par les déchets », Géocarrefour [En ligne], Numéros à venir, mis en ligne le 
05 juillet 2019, consulté le 17 novembre 2020. URL : http://journals.openedition.org/geocarrefour/12663

23. Machikou, N. (2010). Les chemins d'un Etat observateur. Contribution des observatoires régionaux de santé. Thèse de doctorat en Science politique. Université de Picardie Jules Verné.

24. Matejka, G., Bouvet, Y., Evens, E., Koulidiati. J., Ngnikam, E., \& Tanawa, E. (2014). Gestion Maîtrisée des déchets solides urbains et de l'assainissement dans les pays en voie de développement, les besoins en études scientifiques et techniques spécifiques et en outils méthodologiques adaptés. Colloque du développement durable à Ouagadougou, Brukina Faso. Disponible en ligne sur: http://www.francophonie.unilim.fr/public/publications/download/Oua gaaufrefren.pdf.

25. Nay, O. (2008). Lexique de science politique. Vie et institutions politiques. Paris : Dalloz.

26. Ngambi, J.R. (2015). Déchets solides ménagers de la ville de Yaoundé (Cameroun): de la gestion linéaire vers une économie circulaire. Thèse de doctorat en Géographie, Maine : Université du Maine.

27. Ngambi, J.R., Tchindjang, M., \& Ndjogui, T. E. (2011). La prolifération des décharges sauvages et leurs impacts socioenvironnementaux dans la ville de Yaoundé. International Journal Advanced Studies and Research in Africa, 2(1), 52-68. Disponible sur : http://www.africascience.org/.

28. Ngikam E. (2013). Etat de lieu de la gestion des déchets au Cameroun : cas de Yaoundé. Atelier de Lomé du 23 au 29 septembre 2013, Lomé.

29. Obe, H-M., \& Brou Ahossi, N. (2019). Impacts de la Décharge Publique d'Akouédo sur le Cadre de Vie et la Santé de la Population Riveraine. European Scientific Journal, 15(12), 20-29.

30. Ortar, N., \& Anstett, E. (2017). Jeux de pouvoir dans nos poubelles. Economies morales et politiques du recyclage au tournant du $\mathrm{XXI}^{\mathrm{e}}$ siècle. Paris : Petra.

31. Sidi Ould, S. (2006). Méthodologie de caractérisation des déchets solides ménagers à Nouackchott (Mauritanie) : Contribution à la gestion des déchets et outils d'aide à la décision. Thèse de Doctorat, Limoges : Université de Limoges.

32. Simon, H.A. (1945). Administrative behavior. A study of decisionmaking processes. New York: The Free Press.

33. Yao-Kouassi, Q.C., \& Gohourou, F. (2018). L’influence de la précollecte des ordures ménagères sur la qualité de la salubrité dans le District d'Abidjan (Côte-d'Ivoire). Déchets Sciences et Techniques. INSA de Lyon, 3-10. 
34. Zittoun, P. (2013). Conclusion / Comment les politiques publiques fabriquent la politique. In P. Zittoun, La fabrique politique des politiques publiques: Une approche pragmatique de l'action publique (pp. 321-328). Paris: Presses de Sciences Po.

35. Zoa, A. S. (1995). Les ordures à Yaoundé. Urbanisation, environnement et politique au Cameroun. Paris : l'Harmattan.

\section{Documents officiels consultés :}

1. Constitution du Cameroun du 16 Janvier 1996 ;

2. Loi $n^{\circ} 2004 / 017$ du 22 juillet 2004 portant orientation de la décentralisation au Cameroun ;

3. Loi $n^{\circ} 2004 / 018$ du 22 juillet 2004 portant règles applicables aux communes ;

4. Loi $n^{\circ} 2004 / 019$ du 22 juillet 2004 portant règles applicables aux régions ;

5. Republic of Cameroon: Compendium of Laws on Decentralisation. Produced jointly by MINATD and French Embassy in Cameroon, Service of Cooperation and Cultural Actions. 